\title{
Use of a flexible choledochoscope for pericardioscopy and drainage of a loculated pericardial effusion
}

\author{
K K S WONG, A K C LI \\ From the Department of Surgery, Chinese University of Hong Kong, Prince of Wales Hospital, Hong Kong
}

In recurrent or persistent pericardial effusion a subxiphoid pericardial window may be used to drain fluid from the pericardial cavity. ${ }^{1-3} \mathrm{~A}$ more permanent form of drainage may be achieved if a combined peritoneal pericardial window is used. ${ }^{4}$

We describe a case in which we could not initially reach the pericardial effusion through a pericardial window because it was loculated posteriorly. Subsequently a flexible choledochoscope was used successfully to locate and drain the effusion. Inspection of the heart and the pericardium was performed at the same time.

\section{Case report}

A 42 year old man with adenocarcinoma of the right lung and malignant pericardial and pleural effusions was referred to our department for further management. About $600 \mathrm{ml}$ of pericardial fluid had been drained six days earlier by pericardiocentesis through a subxiphoid approach. It recurred rapidly and in view of his good general condition a subxiphoid pericardial window was created.

At operation, the anterior and inferior surfaces of the heart were found to be loosely adherent to the pericardium. After separation of the heart from the pericardium with blunt dissection the pericardial effusion could not be located.

A flexible bronchoscope (Olympus BF B3R), sterilised by immersion in $2 \%$ glutaraldehyde (Cidex) for three hours, was introduced through the pericardial window in an attempt to visualise the pericardial cavity and locate the effusion. The attempt was unsuccessful because whenever suction was applied through the bronchoscope the pericardium became stuck to the tip and obscured the viewing area. A flexible choledochoscope (Olympus CHF 4B), sterilised by immersion in $2 \%$ glutaraldehyde for eight hours, overcame this problem. With a stream of normal saline flowing from the tip of the choledochoscope, the heart was separated from the pericardium and an excellent view of the pericardial cavity was obtained. Loose adhesions that were present were gently broken down by flexing the tip of the choledochoscope to them. Whenever difficulty was encountered in breaking the adhesions or bleeding occurred, the tip of the choledochoscope was manipulated to a different area in search of another path.

Address for reprint requests: $\operatorname{Dr} \mathrm{K}$ K S Wong, Department of Surgery, Chinese University of Hong Kong, Prince of Wales Hospital, Shatin, Hong Kong.

Accepted 4 November 1986
Eventually the loculated effusion was reached when the tip of the choledochoscope entered a pool of yellowish fluid which drained out of the window because of the increased pressure. The flushing of normal saline through the choledochoscope was stopped and about $100 \mathrm{ml}$ of pericardial fluid was aspirated from the loculus. The choledochoscope was then withdrawn and a size $26 \mathrm{~F}$ Argyle catheter was inserted into the loculus along the path created. A total of $800 \mathrm{ml}$ of pericardial fluid was eventually drained.

The postoperative chest radiograph confirmed a considerable reduction in the size of the cardiac shadow. No evidence of myocardial damage or other complication followed from this procedure. Although an occasional ectopic heart beat was noted during manipulation of the choledochoscope, the patient remained in sinus rhythm and tolerated the procedure well.

\section{Discussion}

The investigation of a patient with pericardial effusion of unknown aetiology includes pericardiocentesis ${ }^{5}$ and sometimes the creation of a pericardial window. ${ }^{6-7}$ Pericardial fluid obtained in both procedures can be sent for cytological, nicrobiological, and biochemical analyses. Biopsy of the pericardium can also be undertaken through the pericardial window. These two procedures have their limitations, however. We cannot inspect the pericardium during pericardiocentesis and a pericardial window will allow only a limited view of the pericardial cavity and the heart. Hence the use of a flexible endoscope to visualise the whole pericardial cavity can be of great benefit in the management of patients with pericardial diseases.

We have shown that the flexible choledochoscope can be used in this situation without any complication. Besides inspection of the pericardium, the choledochoscope allowed us to localise and drain a loculated pericardial effusion. Thus the procedure has both diagnostic and therapeutic applications. In our patient loculation of the effusion probably occurred because the earlier pericardiocentesis caused adhesions to develop between the anterior and inferior surfaces of the heart and the pericardium. The choledochoscope could conceivably be used to perform biopsies of lesions on the pericardium, although this was not necessary in our patient. We believe that pericardioscopy as performed may be useful in the investigation and treatment of pericardial conditions.

\section{References}

1 Hankins JR, Satterfield JR. Pericardial window for malignant pericardial effusion. Ann Thorac Surg 1980;30:465-71. 
2 Levin BH, Aaron BL. The subxiphoid pericardial window. Surg Gynecol Obstet 1982;155:804-6.

3 Ghosh SC, Larrien AJ, Ablaza S, Grana VP. Clinical experience with subxiphoid pericardial decompression. Int Surg 1985; 70:5-7.

4 Dabir R, Warren SE. Drainage of pericardial effusion using the peritoneal and pericardial window technique. Surg Gynecol Obstet 1984;159:485-6.

\section{Book notices}

The Anaerobic Threshold: Physiological and Clinical Significance. L Tavazzi and PE di Prampero (Pp 155; f65.) Basel: S Karger, 1986. ISBN No 3-8055-4395-6.

This book provides an account of an international symposium on the clinical value of the anaerobic threshold in heart and lung disease, held in Veruno, Italy, in late 1985. The papers range from a series of accounts of basic cardiorespiratory and metabolic responses to exercise from senior contributors in the area to reports of attempts to apply the assessment of the anaerobic threshold to evaluate the existence and severity of cardiovascular disease. The book is stated to be suitable for both scientists and physicians in everyday practice, but I fear that the former may be disappointed. Although the basic physiology of the cardiorespiratory responses to exercise and the threshold at which appreciable anaerobic metabolism occurs are elegantly covered in the first five contributions, the remaining papers represent attempts to produce tests that will generate parameters useful for clinical evaluation. The book is likely to be of most use to those concerned in the assessment of exercise tolerance in patients with cardiovascular, respiratory, and metabolic disorders, because the comprehensive accounts of the underlying physiology are combined with reports of efforts to assess these functions in various types of patient. The papers reporting the use and reliability of the assessments of anaerobic threshold, however, are somewhat conflicting, especially for patients who have had a myocardial infarction. This book is unlikely to appeal to a wide readership but it may be of some use for the background information it provides, rather than for providing definitive methods for assessing patients. The excellent accounts of basic physiology could be obtained from other texts costing much less than this one, and those who do buy the book will be a little irritated by the occurrence of spelling and typographical errors, although these are compensated for by the general high quality of the illustrations.-IAM
5 Treasure T, Cotter $\mathrm{L}$. How to aspirate the pericardium. $\mathrm{Br} \mathrm{J}$ Hos Med 1980;24:488-9.

6 Prager RL, Wilson CH, Bender HW. The subxiphoid approace to pericardial disease. Ann Thorac Surg 1982;34:6-9.

7 Little AG, Kremser PC, Wade JL, Levett JM, De Meester TR Skinner DB. Operation for diagnosis and treatment of pericardial effusion. Surgery 1984;96:738-44.
Diagnostic Bronchoscopy: A Teaching Manual. 5th ed. Peter Stradling. (Pp 182; £45.) Edinburgh: Churchill Livingstone 1986. ISBN No 0443033005.

This book continues to succeed superbly well as an intro duction to bronchoscopy. In the five years between this neve edition and its predecessor there has, however, been rela $\propto$ tively little new development in bronchoscopic technique. I $\vec{\delta}$ a new edition justified? The importance of the fibrescope has now, somewhat belatedly, been acknowledged, with the chapter on fibreoscopy extensively revised and now place ahead of the chapter on rigid bronchoscopy. A sensible sec tion has been added about control of bleeding during fibreoscopy, which at last recognises the difficulty in carrying out the usual advice to use the bronchoscope as a plug. The 218 photographs of the bronchoscopic appearances of both normality and pathology are of excellent quality and range and are justifiably the reason for the book's pre-eminence in the subject. Of about 30 new plates, however, only a few make an important addition, those of hamartoma and endo옥 bronchial tuberculosis being the most useful; I was surprised that AIDS related pathology was omitted. I was taught to perform a bronchoscopy facing the patient and so, when was learning, all the plates were the "wrong way up"-leading to curious looks from others watching me read the book upside down. There is no way round this problema however, and indeed it may assist in the development of the

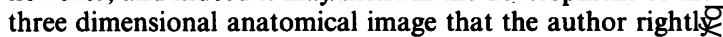
emphasises is important. Given the introductory nature of the text, there are few important omissions. It would have been helpful to include advice on performing bronchoscop 9 in patients with AIDS, to have the question of sedationo discussed more fully, and to have suggestions for furtheō reading. These are relatively minor points, however, and while I would not advise those already owning the fourth edition to rush out and buy this fifth edition, the book re mains an essential companion to those learning the tech nique of bronchoscopy.-IDAJ 